\title{
AN INITIATIVE FOR CONSTRUCTION OF NEW-GENERATION LUNAR GLOBAL CONTROL NETWORK USING MULTI-MISSION DATA
}

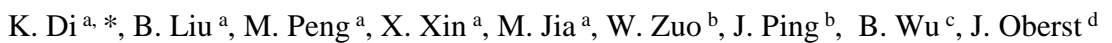

\author{
${ }^{a}$ State Key Laboratory of Remote Sensing Science, Institute of Remote Sensing and Digital Earth, Chinese Academy of \\ Sciences, Beijing, China - (dikc, liubin, pengman, wanwh, xinxin, jiamn)@ radi.ac.cn \\ ${ }^{\mathrm{b}}$ National Astronomical Observatories, Chinese Academy of Sciences, Beijing, China - (zuowei, jsping)@nao.cas.cn \\ ${ }^{c}$ Department of Land Surveying and Geo-Informatics, The Hong Kong Polytechnic University, Hung Hom, Hong Kong \\ - bo.wu@polyu.edu.hk \\ ${ }^{\mathrm{d}}$ Institute for Geodesy and Geoinformation Science, Technical University of Berlin, Berlin, Germany - \\ juergen.oberst@tu-berlin.de
}

Commission III, ICWG III/II

KEY WORDS: Lunar Control Network, Multi-mission Data, Orbital Imaging, Laser Altimetry, Photogrammetric Solution

\begin{abstract}
:
A lunar global control network provides geodetic datum and control points for mapping of the lunar surface. The widely used Unified Lunar Control Network 2005 (ULCN2005) was built based on a combined photogrammetric solution of Clementine images acquired in 1994 and earlier photographic data. In this research, we propose an initiative for construction of a new-generation lunar global control network using multi-mission data newly acquired in the $21^{\text {st }}$ century, which have much better resolution and precision than the old data acquired in the last century. The new control network will be based on a combined photogrammetric solution of an extended global image and laser altimetry network. The five lunar laser ranging retro-reflectors, which can be identified in LROC NAC images and have cm level 3D position accuracy, will be used as absolute control points in the least squares photogrammetric adjustment. Recently, a new radio total phase ranging method has been developed and used for high-precision positioning of Chang'e-3 lander; this shall offer a new absolute control point. Systematic methods and key techniques will be developed or enhanced, including rigorous and generic geometric modeling of orbital images, multi-scale feature extraction and matching among heterogeneous multi-mission remote sensing data, optimal selection of images at areas of multiple image coverages, and large-scale adjustment computation, etc. Based on the high-resolution new datasets and developed new techniques, the new generation of global control network is expected to have much higher accuracy and point density than the ULCN2005.
\end{abstract}

\section{INTRODUCTION}

As a realization of the lunar reference system, a lunar global control network (LGCN) provides geodetic datum and control points for mapping of the lunar surface, and it is of fundamental importance for both scientific and engineering applications. In history, earth-based telescope observations had been used to establish some local or regional lunar control point networks (i.e., catalogs of landmarks of accurately known coordinates) (Schimerman, 1973). Since late 1960s, lunar control networks have been established by photogrammetric solutions using images taken by orbiting spacecraft. Well-known global lunar control networks include the Unified Lunar Control Network (Davies et al., 1994), the Clementine Lunar Control Network (Edwards et al., 1996), and the Unified Lunar Control Network 2005 (ULCN 2005) (Archinal et al., 2006). The widely used ULCN2005 was built based on a combined photogrammetric solution of 43,866 Clementine images acquired in 1994 and earlier photographic data; the resultant 3D positions of 272,931 points have horizontal accuracy of $100 \mathrm{~m}$ to few hundred meters and vertical accuracy of $100 \mathrm{~m}$ level (Archinal, et al., 2006; 2007).
Since the beginning of the $21^{\text {st }}$ century, many nations/organizations have successfully launched new lunar exploration missions, ushering in a new golden age of lunar exploration. Orbital missions, such as European Space Agency's SMART-1, Japan's SELENE (Kaguya), India's Chandrayaan-1, the United States' Lunar Reconnaissance Orbiter (LRO), and China's Chang'e-1 and Chang'e-2 missions, have acquired large volumes of high resolution images and high precision laser altimetry data. In addition, China's Chang'e-3 lander and rover successfully landed and conducted detailed in-situ investigation of the landing site (Liu et al., 2015b).

For comparative and synergistic use of the lunar remote sensing data from multiple missions so that to obtain maximum value for science and exploration, the data sets must be co-registered in a common coordinate reference frame (Kirk et al., 2012). A global lunar control network directly supports such coregistrations. Due to the various uncertainties of the orbits and the imaging sensors, there exist widespread spatial inconsistencies among these new high-resolution data. There also exist considerable differences between the new lunar remote sensing data and ULCN2005. It is highly desirable to construct a new-generation LGCN using the newly acquired

\footnotetext{
* Corresponding author.
} 
multi-mission high-resolution data, so that to better support lunar scientific research and future lunar exploration missions.

Comparing with the data sets used to construct ULCN2005, the new data sets, if used in combination, are significantly better in terms of image resolution, stereo coverage, laser altimetry precision and point density, and orbit precision. For example, benefiting from lunar gravity field data by the GRAIL mission (Zuber et al., 2013), LRO orbit determination reached an accuracy of $\sim 20 \mathrm{~m}$; the accuracy was further improved to $\sim 14 \mathrm{~m}$ after incorporating crossovers of Lunar Reconnaissance Orbiter Laser Altimeter (LOLA) data (Mazarico et al., 2012). Furthermore, the five lunar laser ranging retro-reflectors (LRRRs), which have $\mathrm{cm}$ level 3D position accuracy, can be identified in high resolution images, such as Lunar Reconnaissance Orbiter Camera (LROC) Narrow Angle Camera (NAC) images (Wagner et al., 2012; Liu et al., 2015a). Therefore, these LRRRs can serve as absolute control points in construction of the new LGCN. All these new capabilities make it feasible to construct a new-generation LGCN with much higher accuracy than that of ULCN2005 and the historical ones.

In this research, we propose an initiative for construction of a new generation LGCN using multi-mission data acquired in the $21^{\text {st }}$ century. The data to be used, technical framework and key techniques will be elaborated and discussed in the following sections.

\section{DATA TO BE USED}

Orbital images and laser altimetry data acquired by multiple missions will be used in construction of the new generation LGCN. Their characteristics and contributions to the network are elucidated below.

\subsection{High resolution imagery data}

2.1.1 Chang'e-2 stereo images: Launched on October 1, 2010, the Chang'e-2 (CE-2) orbiter carried a high-resolution CCD stereo camera, which acquired images with a spatial resolution of $7 \mathrm{~m}$ and $1.05 \mathrm{~m}$ respectively at the flight heights of $100 \mathrm{~km}$ and $15 \mathrm{~km}$ (Zhao et al., 2011). The CE-2 CCD camera consists of two line arrays that are separately fixed on the same focal plane, thus offering forward- and backward-looking stereo images (viewing angle 7.98 and -17.2 degrees respectively) in the same track through push-broom imaging. The two line arrays share the same optical axis with a focal length of 144.4 $\mathrm{mm}$. Each line array has 6144 pixels. By completion of the mission, the CE-2 CCD camera obtained 607 orbits of image data, with $7 \mathrm{~m}$ resolution images covering the entire moon and $1.05 \mathrm{~m}$ resolution images covering the preselected landing site of Chang'e-3 (Zuo et al., 2014). Up to now, CE-2 CCD image dataset is the highest resolution stereo image dataset in the world that covers the entire moon.

In previous research, Di et al. (2014) developed a selfcalibration bundle adjustment method that can eliminate the inconsistencies of CE-2 CCD images (back-projection image residuals between images of the same track and neighboring tracks) from more than 20 pixels to subpixel level and also reduce the differences between CE-2 data and LOLA data by 9 $10 \mathrm{~m}$. Li et al. (2015) have been working on the production of new global topographic mapping products, i.e., digital elevation model (DEM) and digital orthophoto map (DOM), using selected 384 imagery strips; comparing with the LRRRs, the planimetric displacement of the CE-2 products is $21 \mathrm{~m}-97 \mathrm{~m}$, the height difference is $2 \mathrm{~m}$ - 19m; comparing with LOLA DEM, the average of height difference is $43 \mathrm{~m}$, standard deviation is $110 \mathrm{~m}$.

Due to its high resolution and coverage, the CE-2 CCD stereo image dataset will be one of the major data sources in construction of the new generation LGCN.

2.1.2 LROC NAC images: NASA's LRO was launched in June 2009 and inserted into a circular (30-50-km), polar orbit. LROC consists of a wide-angle camera (WAC) and two narrowangle cameras (Robinson et al., 2010). WAC has a spatial resolution of $100 \mathrm{~m}$ and swath of $100 \mathrm{~km}$, and NAC acquired images with a spatial resolution of $0.5-2 \mathrm{~m}$ and a swath of $5 \mathrm{~km}$. Using data from the wide-angle camera, a near-global terrain model "GLD100" with a resolution of $100 \mathrm{~m}$ has been produced (Scholten et al., 2012).

LROC NAC images have been widely used for 3D mapping of the priority sites, e.g., past and future landing sites. So far, LROC NAC images almost cover $99 \%$ of the moon surface, but stereo coverage is only 4\% (I. Haase and M. Henrikson, pers. comm., April. 2017). 2D global image mosaics of LRO NAC images have been produced and can be accessed through the Lunaserv mapserver, using the "LROC NAC overlay" feature (Estes et al., 2013). Due to its highest resolution among all lunar orbital images, LRO NAC images will be an important data source in construction of the new LGCN.

2.1.3 SELENE terrain camera images: SELENE (Kaguya) was launched in September 2007 and carried Terrain Camera (TC) consisting of two line arrays for stereo imaging. It acquired images covering over $99 \%$ of the lunar surface with a resolution of $10 \mathrm{~m}$ from the nominal altitude of $100 \mathrm{~km}$. Haruyama et al. (2012) corrected the models of TC detector distortion and attachment angles and reduced the differences between TC DEMs and the laser altimeter measurements; subsequently, global DEM and DOM were produced and released with a resolution of 1024 pixels per degree $(30 \mathrm{~m} /$ pixel at the equator). SELENE TC stereo images have slightly lower resolution that that of CE-2 stereo images, and can be used complementarily in this work.

\subsection{Laser altimetry data}

Launched in June 2009, LOLA acquires 5 parallel profiles, separated by $\sim 56 \mathrm{~m}$; shots along the track are $\sim 10-12 \mathrm{~m}$ apart The range resolution of LOLA data is $10 \mathrm{~cm}$ (Smith et al., 2010a). LOLA DEMs with different resolution have been produced and released, e.g., a global DEM with a resolution of 256 pixels per degree $(118 \mathrm{~m} /$ pixel at the equator $)$ was produced using 6.5 billion LOLA measurements gathered between July 2009 and July 2013 (Smith et al., 2010b). Currently, the LDEM_1024 is the highest resolution global DEM with a resolution of 1024 pixels per degree $(30 \mathrm{~m} / \mathrm{pixel}$ at the equator) (Smith et al., 2010b).

Recently, Barker et al. (2016) produced a lunar DEM by coregistration and combining SELENE TC DEM with LRO laser altimetric data. The model, designated as SLDEM2015, covers latitudes within $\pm 60^{\circ}$, at a horizontal resolution of 512 pixels per degree ( $\sim 60 \mathrm{~m}$ at the equator) and a 3 to $4 \mathrm{~m}$ root-meansquare (RMS) elevation residuals to LOLA profiles.

As of March 2016, LOLA has obtained $~ 6.8$ billion altimetric measurements and will continue to acquire high precision 
altimetric points (Smith et al., 2017). The LOLA data will be one of the major data sources in construction of the new generation LGCN.

\subsection{Absolute control points}

It is critical and beneficial to have some absolute control points in image block adjustment to ensure high accuracy. The five LRRRs, established in missions of Apollo 11, 14 and 15, and the Lunokhod 1 and 2 missions, reach $\mathrm{cm}$ level accuracy through long-term measurements and can be treated as absolute control points (Wagner et al., 2012). Traditionally, it was very hard to incorporate such absolute control points in lunar mapping and establishment of lunar control network because the image resolutions are not sufficient to identify these LRRRs. With the advent of high resolution images, particularly the up to $0.5 \mathrm{~m}$ resolution LRO NAC images, these LRRRs are clearly discernible (Wagner et al., 2012; Liu et al., 2015a). This makes it feasible and attractive to incorporate these LRRRs as absolute control point in construction of the new LCGN.

Recently, a new radio total phase ranging method has been developed and used for high-precision positioning of Chang'e-3 lander; this shall offer a new absolute control point (Ping, 2016).

\section{TECHNICAL FRAMEWORK AND KEY TECHNIQUES}

\subsection{Overall framework}

The new control network will be based on a combined photogrammetric solution of an extended global image and laser altimetry network. The five LRRRs and Chang'e-3 lander will be used as absolute control points in the global adjustment. The laser altimetry data will be used as vertical control. Figure 1 shows the overall technical framework for construction of the new generation LGCN. Key techniques are explained and discussed in the following sub-sections.

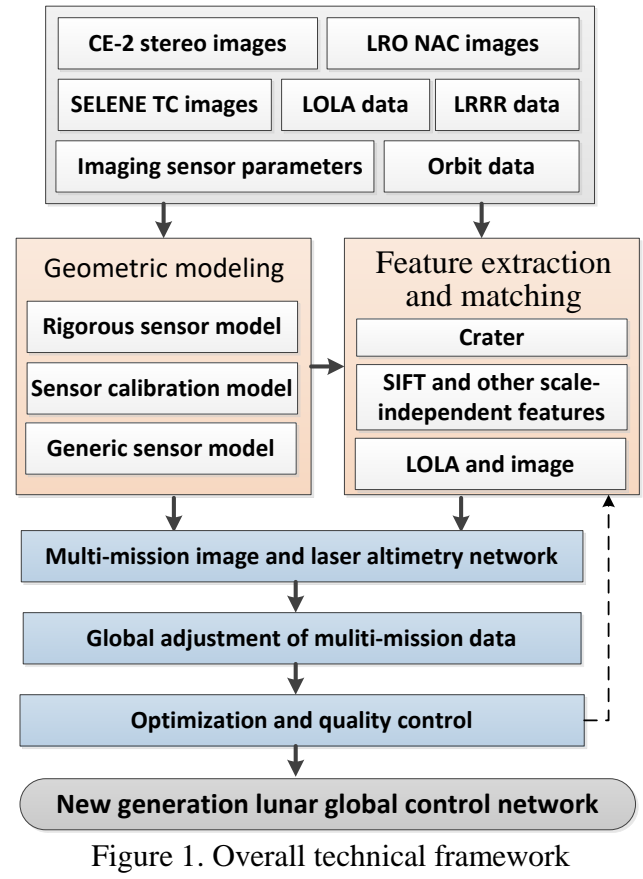

This contribution has been peer-reviewed.

\subsection{Key techniques}

Systematic methods and key techniques will be developed, enhanced and integrated, including rigorous and generic geometric modeling of orbital images, multi-scale feature extraction and matching among heterogeneous multi-mission remote sensing data, optimal selection of images at areas of multiple image coverages, and large-scale adjustment computation, etc.

\subsubsection{Geometric modeling of orbital images: Geometric} modeling of the multi-mission orbital images is the basis for photogrammetric processing of these images. Rigorous sensor models of the orbital images have been established by different groups of researchers based on collinearity equations with exterior orientation (EO) and interior orientation (IO) parameters (Tran et al., 2010; Haruyama et al., 2012; Di et al., 2014; Speyerer et al., 2016; Wu et al., 2014, 2017).

IO refers to the transformation from image coordinate system (lines and samples) to the focal plane coordinate system centred at the principal point of the image according to the calibrated IO parameters of the camera. Due to the complexity and difference in design of the imaging sensors, the IO processes for different sensors are often different, e.g., there may be different numbers of IO parameters and the coordinate transformation equations may be different.

EO refers to the coordinate transformation from the focal plane coordinate system to an object space coordinate system, e.g., lunar body-fixed coordinate system. For push-broom imaging sensors, each image line has a different set of EO parameters. The changes of the EO parameters over short trajectories are usually modelled using polynomials.

The rigorous sensor models are generally complex and different for different sensors. It is preferable to be used in bundle adjustment of images from the same sensor of the same mission. But it would be very complex to use rigorous sensor models in adjustment of images from multi-missions.

Rational function model (RFM) is a commonly used generic geometric model in photogrammetric processing of highresolution earth observation images. It is a mathematical fitting of rigorous geometric model, and has advantages of platform independence, simple form, and high calculation speed (Di et al., 2003; Liu et al., 2011). Recently, Liu et al. (2014, 2016) studied the feasibility and accuracy of RFM fitting for lunar (LRO NAC, CE-1, CE-2) and Mars (HiRISE, HRSC) orbital images. Experimental results show that the traditional line-based RFM can precisely fit the rigorous sensor models with a RMS residual of 1/100 pixel level for orbiters without exposure time changing; while for orbiters with exposure time changing (e.g., CE-2 and HRSC), the proposed two solutions, time-based RFM or sensor corrected images with line-base RFM, can also reach such a high fitting precision (Liu et al., 2016).

3.2.2 Multi-mission image and laser altimetry network: An extended global image and laser altimetry network is essential to a high-precision photogrammetric solution for the LGCN. The network should consists of sufficient number of evenly distributed tie points (homologous feature points) that link all the images together based on feature extraction and matching among multi-mission remote sensing data. Due to the differences in image resolution (e.g., $0.5 \mathrm{~m}$ of LRO NAC vs. $7 \mathrm{~m}$ of $\mathrm{CE}-2$ ), pixel aspect ratio, pointing angle, illumination 
condition, etc., extraction and matching features among multimission images are very challenging. We propose feature extraction and matching among heterogeneous multi-mission remote sensing data using crater, scale-invariant feature transform (SIFT) and other scale-invariant features.

Impact craters are the most common morphologic landforms on the Moon. Many automated methods have been developed to detect and extract craters from lunar images and/or DEMs (e.g., Kang et al., 2015). Bowl-shaped simple craters can be extracted from images with different resolutions using these methods with some manual checking and editing; the coordinates of the centres and sizes (i.e., radii) of the craters are then obtained through least squares fitting. Matching of craters between images can be done based on distribution pattern matching with RANSAC-like outlier detection. As a result, images with different resolutions from different mission are matched and the craters centres are used as tie points to build the image network.

SIFT is a popular algorithm to detect and describe local features, and is invariant to image scale and rotation (Lowe, 2004). Matching of SIFT features (keypoints) is realized using Euclidean distance of their feature vectors. SIFT matching can be applied as a complementary to crater matching in image areas where there are few craters. Other scale-invariant features, such as SURF (Bay et al., 2008) and AKAZE (Alcantarilla et al., 2013) features, will be compared with SIFT and can be used combinedly to extract and match more features.

Matching between image and altimetry data poses another challenge due to their heterogeneous natures. One practical way is to use DEM as a "bridge" between the 3D laser points and the image. The LOLA DEM, or the LOLA+TC merged DEM, can be used to generated simulated images using hill-shading techniques with the sun azimuth and elevation angles same as that of the images to be matched. Then, the simulated images are comparable to the actual images, and the image matching methods described above can be applied. Consequently, the altimetry points are matched to the images.

\subsubsection{Optimal selection of images in areas of multiple} image coverages: With continual data acquisition by multiple lunar orbiter missions, many areas of the lunar surface have been covered many times by different orbiters or the same orbiter. Optimal selection of images is very important in order to achieve the best geopositioning precision in those areas with multi-image coverages.

In order to automatically identify stereo image pairs for topographic mapping, Becker et al. (2015) provide recommended methods and criteria considering image overlap, 3D stereo imaging "strength", as well as similarity in spatial resolution, illumination, and spectral wavelength range.

Recently, we performed an empirical analysis of the geopositioning precision of multi-image triangulation using LROC NAC images at the CE-3 landing site. Experiments with multiple images indicate that utilizing more images produces higher precision than almost all dual-image models; meanwhile, using fewer images can produce better precision than using all available images together ( $\mathrm{Di}$ et al., 2016). A progressive selection method has been proposed to find the best image combination for maximum precision. With further validation and improvements, the method can be used in optimal selection of images in construction of the image network.
In some special cases, spacecraft vibrations can cause angular "jitter" and image distortions. Images with jitter effects should be avoided in image selection for control network construction.

3.2.4 Global adjustment of multi-mission data: Global combined adjustment of the multi-mission image and laser altimetry network is the key to the success of the new generation LGCN. Considering the complexities and differences of multiple imaging sensors, we propose to perform global adjustment of multi-mission data based on the generic sensor model - RFM. The rational polynomial coefficients (RPCs) of the RFM are computed by fitting of the rigorous sensor models of the images of different sensors. To ensure the fitted RPCs are sufficiently accurate, we will perform regional bundle adjustment to improve the accuracy of the EO parameters of the participating images.

For regional bundle adjustment, the lunar globe is divided into to regional image blocks with some overlaps between neighboring blocks, and each block consists of multiple strips of images. For each block, images from different missions (i.e., CE-2, SELENE) will be adjusted separately based on rigorous sensor models with LOLA data as vertical constraints. Combined block adjustment of CE-2 stereo images (or SELENE images) and LOLA data can be achieved using the method developed in $\mathrm{Wu}$ et al. (2014) to improve the EO parameters and can also incorporate self-calibration parameters to improve IO parameters (Di et al., 2014). Since we use LOLA data as a reference in the regional bundle adjustment and since the LRO orbit has been refined, it is not necessary to re-adjust the EO parameters of the LRO NAC images in this stage. But the geometric calibration results (Speyerer et al., 2016; Wu et al., 2017) should be considered when generating RPCs for NAC images.

After the regional adjustment, the EO and IO parameters of the participating images are refined such that the inconsistencies between neighboring image strips are eliminated/reduced, and the $3 \mathrm{D}$ coordinates of the tie points are more consistent with the LOLA data. Based on the refined EO and IO parameters of the images, RPCs of the images will be obtained through least squares fitting (Di et al., 2003, Liu et al., 2016).

Inconsistencies among multi-mission images, typically adjusted separately, will also be furthered reduced in the global adjustment. According to previous research and experiments, the regional adjustment should be able to reach a sup-pixel accuracy in image space; considering that the LRO orbit has been refined to an accuracy of $14 \mathrm{~m}$ and the LOLA data is used as reference, an accuracy of within $20 \mathrm{~m}$ in object space (i.e., the lunar body fixed frame) can be expected for the regional adjustment.

The global adjustment of the multi-mission image and laser altimetry network is based on a combined photogrammetric solution. Three types of tie points (intra-strip tie point linking the stereo images of the same strip, inter-strip tie points linking neighboring images of the same sensor, and inter-sensor tie point linking images from different sensors) will be used in the global adjustment. Weights for different observations (tie point measurements) from multi-mission data will be determined according to their a priori standard deviations, which are related to feature matching accuracy and image resolution. The unknowns for the global adjustment include the 3D ground positions of the tie points and the correction parameters (e.g., affine parameters in image space) for the RPCs. The five 
LRRRs will be used as absolute control points. The LOLA data will be used as vertical constraints in a way that the ground positions of the image tie points obtained through multi-image triangulation using the image EO parameters should be consistent with a local surface determined by the nearby LOLA points.

It is an interesting issue whether the LOLA points should also be adjusted in the global adjustment, as well as in the regional adjustment. In previous research, adjustment of LOLA point was done by back-projecting the LOLA points onto the images using the sensors models and taking the projected image points as observations; but due to their high accuracy, the LOLA points were only slightly adjusted in local areas (Wu et al., 2014). For a global adjustment, this would significantly increase the computing effort. We will further study the necessity and effectiveness of adjusting LOLA points in the photogrammetric adjustment.

Comparing with the regional adjustment, the global adjustment further refines the $3 \mathrm{D}$ coordinates and the image model parameters in a global optimization manner and with absolute control from LRRRs. We expect the accuracy of the resultant tie points can reach $20 \mathrm{~m}$ to $30 \mathrm{~m}$.

In computation point of view, the global adjustment is the process of solving large-scale matrix equations. Sparse matrix technique is necessary to improve the efficiency of the solution.

As part of the outputs of global adjustment, adjusted 3D ground coordinates of the tie points, along with their image coordinates in the related images, will be gathered to form the new generation of LGCN. More matched feature points can be used to densify the LGCN; their ground coordinates are calculated by multi-image triangulation (space intersection) using the adjusted image model parameters, i.e., RPCs and the correction parameters. As a result, the accuracy and point density of the new LGCN should be much higher than those of the ULCN2005.

\section{CONCLUDING REMARKS}

With the availability of huge volumes of high resolution images and altimetry data covering the entire lunar surface, and with the development of new photogrammetric techniques, it is both desirable and feasible to construct a new generation lunar global control network using high resolution data newly acquired by multiple missions in the $21^{\text {st }}$ century. In this initiative, we proposed a technical framework, described the relevant data, elaborated and discussed the key techniques for construction of a new generation LGCN.

To realize this initiative not only requires enhancement and integration of advanced photogrammetric techniques, but also involves massive data processing work. It requires considerable funding to support multiple teams to work together for the common goal. International collaboration is particular important and indispensable for construction of the new LGCN using multi-mission data.

\section{ACKNOWLEDGMENTS}

This study was supported in part by National Natural Science Foundation of China under Grants 41671458 and 41590851.

\section{REFERENCES}

Alcantarilla, P.F., J. Nuevo, and A. Bartoli, 2013. Fast Explicit Diffusion for Accelerated Features in Nonlinear Scale Spaces. Proceedings British Machine Vision Conference (BMVC), Bristol, UK, pp. 13.1-13.11.

Archinal, B.A., M.R. Rosiek, R.L. Kirk, B.L. Redding, 2006. The unified lunar control network 2005. USGS open-file report. http://pubs.usgs.gov/of/2006/1367/ULCN2005-OpenFile.pdf

Archinal, B.A., M.R. Rosiek, R.L. Kirk, T.L. Hare, B.L. Redding, 2007. Final Completion of the Unified Lunar Control Network 2005 and Topographic Model. 38 ${ }^{\text {th }}$ Lunar and Planetary Science Conference, League City, Texas, p.1904.

Barker, M.K., E. Mazarico, G.A. Neumann, et al., 2016. A new lunar digital elevation model from the Lunar Orbiter Laser Altimeter and SELENE Terrain Camera. Icarus, 273, pp. 346355

Bay, H., A. Ess, T. Tuytelaars, L. Van Gool, 2008. SURF: Speeded Up Robust Features. Computer Vision and Image Understanding (CVIU), 110(3), pp. 346-359.

Becker., K. J., B. A. Archinal, T. M. Hare, R. L. Kirk, E. Howington-Kraus, M. S. Robinson and M. R. Rosiek, 2015. Criteria for Automated Identification of Stereo Image Pairs. In: Lunar and Planetary Science Conference, Texas, USA, 46, pp. 2703.

Di, K., R. Ma, and R. Li, 2003. Rational Functions and Potential for Rigorous Sensor Model Recovery. Photogrammetric Engineering and Remote Sensing, 69(1), pp. 33-41.

Di, K., Y. Liu, B. Liu, M. Peng and W. Hu, 2014. A Selfcalibration bundle adjustment method for photogrammetric processing of Chang'E-2 stereo lunar imagery. IEEE Transaction on Geoscience and Remote Sensing, 52(9), pp. 5432-5442.

Di, K., B. Xu, B. Liu, M. Jia, Z. Liu, 2016. Geopositioning precision analysis of multiple image triangulation using LRO NAC lunar images. Proceedings of 23rd ISPRS Congress, Commission IV, 12-19 July, Prague, Czech Republic, PP. 369374.

Davies M.E., et al. 1994. The unified lunar control network: 1994 version. Journal of Geophysics Research, 99(E11), pp. 23211-23214.

Edwards K.E., et al. 1996. Global Digital Mapping of the Moon. $27^{\text {th }}$ Lunar and Planetary Science Conference, pp. 335-336

Estes, N. M., C.D. Hanger, A.A. Licht, E. Bowman-Cisneros, 2013. Lunaserv Web Map Service: History, Implementation Details, Development, and Uses. $44^{\text {th }}$ Lunar Planet. Sci. Conf., abstract \# 2069.

Haruyama, J., S. Hara, K. Hioki, et al., 2012. Lunar global digital terrain model dataset produced from SELENE (Kaguya) terrain camera stereo observations. 43rd Lunar and Planetary Science Conference, abstract \# 1200. 
Kang, Z., Z. Luo, T. Hu and P. Gamba, 2015. Automatic Extraction and Identification of Lunar Impact Craters Based on Optical Data and DEMs Acquired by the Chang'E Satellites. IEEE Journal of Selected Topics in Applied Earth Observations and Remote Sensing, 8(10), pp. 4751-4761.

Kirk, R., B. Archinal, L. Gaddis and M. Rosiek, 2012. Lunar Cartography: Progress In The 2000s and Prospects for the 2010s. In: International Archives of the Photogrammery, Remote sensing and Spatial Information Sciences, B4, pp. 489494.

Li, C. L., X. Ren, J. J. Liu, et al., 2015. A New Global and High Resolution Topographic Map Product of the Moon from Chang'E-2 Image Data. 46th Lunar Planetary Science Conference, abst. \#1638

Liu, B., Y. Liu, K. Di and X. Sun, 2014. Block adjustment of Chang'E-1 images based on rational function model. In: Remote Sensing of the Environment: 18th National Symposium on Remote Sensing of China, International Society for Optics and Photonics, pp. 91580G-91580G.

Liu, B., K. Di, B. Wang, G. Tang, B. Xu, Z. L. and Z. Liu, 2015a. Positioning and precision validation of Chang'E-3 Lander based on multiple LRO NAC images (in Chinese with English abstract). Chinese Science Bulletin, 60, pp. 2750-2757.

Liu, B., B. Xu, K. Di, M. Jia, 2016. A Solution to Low RFM Fitting Precision of Planetary Orbiter Images Caused by Exposure Time Changing. International Archives of the Photogrammetry, Remote Sensing and Spatial Information Sciences, 441-448.

Liu, Y. and K. Di, 2011. Evaluation of Rational Function Model for Geometric Modeling of Chang'E-1 CCD Images. In: International Achieves of the Photogrammetry, Remote Sensing and Spatial Information Sciences, Guilin, China, Vol. 38, Part 4/W25, pp. 121-125.

Liu, Z., K. Di, M. Peng, W. Wan, B. Liu, L. Li, T. Yu, B. Wang, J. Zhou and H. Chen, 2015b. High precision landing site mapping and rover localization for Chang'e-3 mission. Science China-physics Mechanics \& Astronomy, 58(1), pp. 1-11.

Lowe, D.G., 2004. Distinctive image features from scaleinvariant keypoints. International journal of computer vision, 60(2), pp. 91-110.

Mazarico, E., D. D. Rowlands, G. A. Neumann, et al., 2012. Orbit determination of the Lunar Reconnaissance Orbiter. Journal of Geodesy, 86(3), pp. 193-207.

Ping, J. S., 2016. Experiment of Lunar Radio Phase Ranging Using Chang'e-3 Lander. $47^{\text {th }}$ Lunar and Planetary Science Conference, The Woodlands, Texas, p. 1339

Robinson, M., S. Brylow, M. Tschimmel, et al., 2010. Lunar Reconnaissance Orbiter Camera (LROC) instrument overview. Space Science Reviews, 150, pp: 81-124.

Schimerman, L A, 1973. Lunar Cartographic Dossier. Volume I, NASA and the Defense Mapping Agency, St. Louis, Missouri.
Scholten, F., J. Oberst J, K.D Matz, T. Roatsch, M. Wählisch, E. Speyerer, M. Robinson, 2012. GLD100: The near - global lunar $100 \mathrm{~m}$ raster DTM from LROC WAC stereo image data. Journal of Geophysical Research: Planets, 117(E12).

Smith, D.E., M.T. Zuber, G.A. Neumann, et al., 2010a. Initial observations from the Lunar Orbiter Laser Altimeter (LOLA). Geophysical Research Letters, 37, pp. L18204.1-6

Smith, D.E., M.T. Zuber, G.B. Jackson, et al., 2010b. The Lunar Orbiter Laser Altimeter Investigation on the Lunar Reconnaissance Orbiter Mission. Space Science Review, 150, pp. 209-241.

Smith, D. E., M. T. Zuber, G. A. Neumann, et al., 2017. Summary of the results from the lunar orbiter laser altimeter after seven years in lunar orbit. Icarus, 283, pp. 70-91.

Speyerer, E.J., R.V., Wagner, M.S. Robinson, et al., 2016. Preflight and onorbit geometric calibration of the Lunar Reconnaissance Orbiter Camera. Space Sci. Rev., 200, pp. $357-$ 392.

Tran, T., E. Howingtonkraus, B. Archinal, M. Rosiek, S. Lawrence, H. Gengl, D. Nelson, M. Robinson, R. Beyer, R. Li, J. Oberst and S. Mattson, 2010. Generating Digital Terrain Models from LROC Stereo Images with SOCET SET. In: Lunar and Planetary Science Conference, Texas, USA, 41, pp: 2515.

Wagner, R. V., E. J. Speyerer, K. N. Burns, et al., 2012. Revised coordinates for apollo hardware. In: International Archives of the Photogrammetry, Remote Sensing and Spatial Information Sciences, 1, pp. 517-521

$\mathrm{Wu}$, B., H. Hu, and J. Guo, 2014. Integration of Chang'E-2 Imagery and LRO Laser Altimeter Data with a Combined Block Adjustment for Precision Lunar Topographic Modeling. Earth and Planetary Science Letters, 391, pp. 1-15.

Wu, B., and W.C. Liu, 2017. Calibration of Boresight Offset of LROC NAC Imagery for Precision Lunar Topographic Mapping. ISPRS Journal of Photogrammetry and Remote Sensing, 128, pp. 372-387.

Zhao, B., J. Yang, D. Wen, W. Gao, L. Chang, Z. Song, B. Xue, and W. Zhao, 2011. Overall scheme and on-orbit images of Chang E-2 lunar satellite CCD stereo camera. Sci. China Ser. E, Technol. Sci., 54(9), pp. 2237-2242.

Zuber, M. T., D. E. Smith, M. M. Watkins, et al., 2013. Gravity Field of the Moon from the Gravity Recovery and Interior Laboratory (GRAIL) Mission. Science, 339, pp. 668-671.

Zuo, W., C. Li, and Z. Zhang, 2014. Scientific data and their release of Chang'E-1 and Chang'E-2. Chin. J. Geochem., 33, pp. 24-44. 